\title{
Testing the Responses of Three PSF Fitting Methods
}

\author{
Meridith Joyce \\ John Marriner \\ Fermilab
}

August 10, 2012

\begin{abstract}
We present an analysis of prototype code designed to fit point-spread functions to point-like astronomical objects appearing in images taken by DECam, or the Dark Energy Camera. We test three methods, each of which yields a maximum likelihood estimate for a different likelihood function. We call these methods Poisson likelihood, $\chi^{2}$ with background error, and $\chi^{2}$ with data error, and describe their differences mathematically. Each is used to construct a fit involving up to four parameters characterizing the raw pixel data. We measure biases in the signal and background levels under a variety of conditions. We test the variance and error estimate of the fits as a function of signal strength, outline areas of sensitivity, and demonstrate the effects of incomplete or inaccurate information on the Poisson fit. We conclude that the Poisson likelihood fitting method is best, as it is successful in all circumstances under which the $\chi^{2}$ series are, and displays other favorable attributes where the others do not.
\end{abstract}

\section{Introduction}

One of the most important astronomical measurements is a precise determination of the flux of a distant object. Because observations have significant measurement errors, parameter estimation techniques are used to obtain measurements of the flux. One of the applications of photometry is to provide the best estimator of the brightness of astronomical objects that can be determined from images of the sky. One approach to this problem is aperture photometry, in which the brightness of an object is measured by counting photons in a given region of an image after performing background-subtraction. This method is robust because it is insensitive to the shape of the object or its position. However, it is more sensitive to statistical errors. For bright objects, this method is effective, but it is weak statistically, or inefficient, and therefore less precise for faint objects. Another method is PSF photometry. The "point-spread function," or PSF, describes the response of an imaging system when measuring point-like objects. On a digitized image, a point-like object is observed as an array of pixels that forms a 2-dimensional peak around the center of the source; e.g. the point of light is "smeared out," or spread statistically, over a larger region. When we investigate faint stars, which are effectively point sources, the more efficient PSF photometry is preferable, but 
requires attention to the shape and location of the center, which can introduce errors. If the star is faint, the position is uncertain and the estimate for the flux will be biased. We expect a bias in flux when the position is fit because the displacement from the center can only be positive, since it is measured radially. We develop computer code that simulates observations and uses variations on a mathematical function known as the "likelihood function" (see §2) used to estimate up to four parameters characterizing data. We analyze the precision and bias of the program when applied to simulated data. We derive the mathematical techniques and test three fitting methods: Poisson likelihood, chi-squared with background error, and chi-squared with data error, in four parameter systems. We describe the numerical methods used in constructing the fits in section 2 and present graphical comparisons of the methods in sections 4 and 5 . We demonstrate where biases and other errors occur and comment on the effectiveness of each method under various parameter systems and fitting options. We conclude that the Poisson likelihood fitting method is accurate in the largest range of conditions and produces a bias consistent with zero in all cases where there is sufficient signal level.

\section{Numerical Methods}

We describe the mathematics behind the PSF and our fitting methods.

In an image, we assume an observation of a grid of $N \times N$ pixels, where the pixels are numbered from 1 to $N^{2}$ and the $i^{\text {th }}$ pixel observation is $n_{i}$ photoelectrons. The $n_{i}$ are not integer values because of readout noise, and each observation is equal to the average number of photons $\bar{n}_{i}$ plus statistical fluctuations. We further assume that there is a point-spread function (PSF) that describes the distribution of light from a point source that is known with negligible error and is given by the function $\mathrm{P}(\mathrm{x}, \mathrm{y})$. We model the average number of photo-electrons by

$$
\bar{n}_{i}=A P\left(x_{i}-s_{x}, y_{i}-s_{y}\right)+B
$$

where $A$ is the amplitude of the source (amount of signal), $B$ is the level of background counts, and $s_{x}$ and $s_{y}$ give the centering of the PSF.

We can estimate the parameters $A, B, s_{x}$, and $s_{y}$ by finding the values that maximize a likelihood function. We consider two possible approximations to the likelihood function. The first form assumes that the $n_{i}$ follow a chi-squared distribution with error $\sigma_{i}$ (which is approximately true when the $n_{i}$ are large). The likelihood is

$$
\begin{array}{rll}
\mathcal{L}=e^{\left(-\chi^{2} / 2\right)} & \\
-2 \ln (\mathcal{L}) & = & \chi^{2} \\
& = & \sum_{i=1}^{N^{2}}\left[\frac{\bar{n}_{i}}{\sigma_{i}}\right]^{2} .
\end{array}
$$


We implement two fitting techniques based on maximizing this form, and we refer to them as " $\chi^{2}$ with data error" (shortened to " $\chi^{2}$ data" in figures) and " $\chi^{2}$ with background error" (shortened to " $\chi^{2}$ bkg" in figures) henceforth. The difference between them emerges with selection of the error term $\sigma_{i}$. One obvious possibility is to approximate $\sigma_{i}=\sqrt{n_{i}}$, but $\sigma_{i}$ is not the true error because of statistical fluctuations in the data. This is the $\chi^{2}$ data approach. Another common technique is to set the $\sigma_{i}$ to be some constant, say $\sigma_{i}=B$. This is the $\chi^{2}$ background approach. This latter choice is not a very good estimate of the actual errors, but the maximum likelihood is insensitive to the values of the $\sigma_{i}$ and the technique works quite well. A more accurate method would be to set $\sigma_{i}=\sqrt{\bar{n}_{i}}$, but this has the technical disadvantage of placing the fit parameters in the denominator of the function being minimized, making the fit non-linear. However, if we fit for the PSF shift parameters $s_{x}$ and $s_{y}$, the fit is non-linear anyway, so the disadvantage is slight.

Instead of pursuing the option of $\sigma_{i}=\sqrt{\bar{n}_{i}}$, we examine a new approach that should be more general. This alternative form is based on Poisson statistics.

$$
\begin{aligned}
\mathcal{L} & =\prod_{i=1 \mid}^{N^{2}} e^{-\bar{n}_{i}} \frac{\bar{n}_{i}}{n_{i} !} \\
\ln (\mathcal{L}) & =\sum_{i=1}^{N^{2}}-\bar{n}_{i}+\ln \bar{n}_{i}-n_{i} \ln n_{i}+n_{i}
\end{aligned}
$$

where we have used Stirling's approximation

$$
\ln n ! \approx n \ln n-n
$$

. We name the technique based on maximizing this variant "Poisson likelihood," and refer to it as such subsequently. It can be shown that the Poisson likelihood is equivalent to setting $\sigma_{i}=\sqrt{\bar{n}_{i}}$ in Equation 4, when $\bar{n}_{i}$ is large. A disadvantage of the Poisson likelihood method is that the non-linear fit for parameters $A$ and $B$ requires iterative techniques, even when $s_{x}$ and $s_{y}$ are not being fit.

In general, the background will be smooth and the value of $B$ can be estimated from pixels outside the $N \times N$ grid. We can express this information as a prior on $B$ in either the case of Equation 4 or Equation 6 by adding an additional term

$$
-2 \ln \left(\mathcal{L}^{\prime}\right)=-2 \ln (\mathcal{L})+\frac{\left(B-B_{P}\right)^{2}}{\sigma_{B}^{2}}
$$

where $B_{P}$ represents the prior knowledge of $B$ and $\sigma_{B}$ the uncertainty.

\section{Implementation}

We choose to simulate a $25 \times 25$ pixel grid of observations. For most of our tests, the simulated point source is centered on the grid, but we consider situations in which it is not, so the fit can adjust 
for this in these cases by varying parameters $s_{x}$ and $s_{y}$. We construct a PSF on a similar $51 \times 51$ grid, and the PSF is shifted by $s_{x}$ and $s_{y}$ using Lanczos interpolation in the process of fitting for $s_{x}$ and $s_{y}$. The PSF is shifted, scaled, and interpolated to match the data. We implement routines that construct a series of simulated observations based on point-like objects using the PSF, but with added errors due to photon statistics and readnoise. We compare the numbers generated by these functions to "true value" parameters $A, B, s_{x}$ and $s_{y}$ which were used to generate the simulated observations. Our nominal values correspond approximately to DECam exposures of 100 seconds in the $g$-band, and are given by $A=1062, B=742, s_{x}=0$ and $s_{y}=0$. These numbers roughly translate to expecting a detectable signal, moderate background noise, and known centering of the PSF. We use a set of control variables to manipulate the conditions and type of fit. We track the discrepancy between the "true fit," a preexisting correct answer determined by which values are selected for $A, B, s_{x}$ and $s_{y}$, and the fit generated by the computer. Reasons for discrepancies between them include low signal, high background noise, and incorrectly centering the PSF.

We run all simulations in one of four possible parameter systems. We use the variable $n$ to denote which set of parameters is being fit. The first case, denoted by $n=1$, involves fitting only for $A$ (signal only), the second, $n=2$, fits for $A$ and $B$ (signal and background), the third, $n=3$, fits for $A, s_{x}$ and $s_{y}$ (signal and position), and the fourth, $n=4$, fits for $A, B, s_{x}$ and $s_{y}$ (signal, background, and position). If a parameter is not being fit, it is held at its fixed true value, which for $B$ is 742 and for $s_{x}$ and $s_{y}$ is 0 . Every parameter system involves $A$, so it is never automatically forced to a default value. Additionally, we experiment with forced errors and dependence on prior information.

In the main program, a series of arrays is filled with statistical quantities describing a routine's computation. Routines written elsewhere are called to construct data and a Gaussian fit (PSF) to that data from the values assigned to $A$, etc. by the user at onset. Choosing a fitting method corresponds to choosing the way in which the computer will attempt to minimize the difference between the data it generates and the fit it constructs. Two of the options for the fitting method, $\chi^{2}$ background and $\chi^{2}$ data, are based on the likelihood maximization function described in equation (4). The other, Poisson likelihood, is described in equations (5) and (6). The method we call " $\chi^{2}$ data" is a $\chi^{2}$ minimization routine that takes into account error in the generated data; $\chi^{2}$ background is another minimization routine, but takes into account error in the signal background instead. We are interested in assessing the comparative accuracy of these three methods, and do so in the Comparison of Methods section. There we also highlight the advantages of the Poissonlikelihood method.

The majority of data is presented as a function of the signal level, parameter $A$. We collect biases, RMS values, and mean error estimates by calculating fits over a range of $A$ values. We do this by looping over a list of regularly-incremented values and assigning each one, in turn, to $A$, and generating a new set of fit statistics for each outcome. If we are interested in the fit output for various levels of background noise, we will increment $B$, and so forth. For a given set of fits, the parameter NTRIALS controls the computational accuracy of the fit, which is the number of trials 
run for a given set of fitting conditions. In other words, it is the number of trials used in computing a single data point on the figures that follow. "Trial" here is a statistical term, meaning a single set of pseudo-randomly generated numbers, extracted from either a Poisson or Gaussian curve centered at the true parameter value, used in computing the fit. NTRIALS is set to either 1000 or 10,000 for all data shown below, meaning that at least 1000 statistical trials were computed per data point. A set of fit values for $A$ through $s_{x}$, as well as biases, errors, RMS values, and other statistical information, is collected for each trial, averaged over the number of trials, and these averages are taken to be the best estimate of the fit information given a particular set of parameters. Using multiple computations based on numbers near the intended values, taken from a distribution of our selection and weighted by the likelihood of their occurrence, gives statistical robustness to the outcome and serves to better simulate true astronomical data, which can be distorted by a plethora of environmental and technological factors. It is important to note the difference between NTRIALS and the statistic we call "ntrials," which is a measure of the number of attempted fits that succeed for a given run. It is possible that a fit to the data will fail to converge, and if this happens, it is a failed trial. The ratio ntrials:NTRIALS gives the percentage of trials that were successful. All of the data shown below was computed under conditions that produced a $95 \%$ or better success rate. The second fit-manipulating parameter, which we call "sigma" (denoted as $\sigma_{P}$ inEquation8, dictates whether or not prior knowledge, e.g. a pr-existing input, is used in fitting for parameter $B$. The $\sigma_{p}$ value is a way of controlling the level of fluctuation from a known input permitted to occur. A small, positive $\sigma_{p}$ value requires the program to fit $B$ with very little fluctuation from the true value of $B$. A large, positive $\sigma_{p}$ value lowers the impact prior knowledge of $B$ has on the fit, and a negative $\sigma_{p}$ value dictates that no prior knowledge of $B$ be used in fitting $B$.

\section{Comparison of Methods}

We compare the three fitting methods through an analysis of the bias, variance, and error estimate for each of the parameter systems. We show the variance and bias in $A$ as a function of $A$ for each fitting method for the 1,2,3, and 4-parameter cases. Additionally, we present plots of the RMS and error estimates against $A$ for all methods. Deeper analysis of the Poisson case, such as its behavior in a small signal region, is given. NTRIALS values for subsequent figures range from 1000 to 10,000 , and a $\sigma_{p}$ of either 0.1 or -1 (heavy or no prior on $B$, respectively) is given where

relevant, i.e. for the 2- and 4-parameter systems. The initial value of $B$ is supplied by the calling program and is normally set to the true value of $B=742$. NTRIALS and $\sigma_{p}$ are invariant for the data sets on each figure. 


\subsection{Bias}

The bias is defined in our program as the true value of a parameter subtracted from the fit's calculation of that parameter; e.g. we define the bias in $A$ as

$$
\text { fit. } A-A
$$

where "fit. $A$ " is the element of the array containing the computed value of $A$. Biases will depend on which parameters are being fit and which values have priors on them or not. We cover several combinations.

Bias in $A$ as a function of $A$ is plotted in Figures 1 and 2. Figure 1a depicts the parameter system that fits for $A$, and Figure $1 \mathrm{~b}$ shows the system that fits for both $A$ and $B$. Figures $2 \mathrm{a}$ and $2 \mathrm{~b}$ show the fits for the system including $A, s_{x}$, and $s_{y}$, and the system including all four parameters, respectively. Each grid shows the three fitting methods: Poisson likelihood as the dotted series, $\chi^{2}$ with data error as triangles, and $\chi^{2}$ with background error as asterisks. The $\chi^{2}$ fit with data error shows a large bias in the 1 , and 3 - parameter systems. The $\chi^{2}$ data fit may show a slight positive bias in the 2-parameter case, but the bias is significantly smaller in magnitude in the 2 and 4 parameter cases than in the 1 or 3 parameter cases. In all but the $n=1$ and $n=3$ cases, the bias in the $\chi^{2}$ data fit worsens as $A$ increases. We observe that in all cases, the Poisson likelihood and $\chi^{2}$ fit with background error are consistent with zero bias.

The difference in the severity of the biasing of the $\chi^{2}$ data fit between the 1 and 3 versus 2 and 4- parameter systems is due to the fact that the even-numbered parameter systems fit for $B$ and the others do not. Clearly the $\chi^{2}$ data fit is inherently biased, but fitting for $B$ decreases the negative effect.

We measure the bias in $B$ as a function of $A$ for the 2- and 4-parameter systems (as these are the only systems in which a fit for $B$ is calculated). Figure 3 a shows the three fits for $n=2$, and Figure $3 \mathrm{~b}$ for $n=4$. In both systems, the Poisson and $\chi^{2}$ background error fits are consistent with zero bias in $B$. The $\chi^{2}$ data error fit shows a negative bias in both cases.

At $A=0$, we expect the majority of the fitting trials to fail when the centering of the PSF is being computed ( $s_{x}$ and $s_{y}$ are being fit), as there is no signal from which to determine the center of the PSF. For this reason, we do not include data at zero for any of the fits or systems, though a fit could be computed in any parameter systems where $s_{x}$ and $s_{y}$ are not being fit. In Figure 4 , we show the Poisson likelihood fit in the small signal regions for the 3 and 4-parameter systems with denser sampling over the 0-5000 range. We find that as signal strength increases, the bias decays toward zero. All methods show similarly increasing bias decreasing signal strength The biasing in low-signal regions is anticipated because it is harder to fit for position with small signal, and the position error is always positive because it is measured radially.

Figure 5 shows another measure of the biases in parameters $\mathrm{A}$ and $\mathrm{B}$, this time as a function background prior, $B_{P}$. Recall that the purpose of $B_{P}$ is to force an estimate of the background 


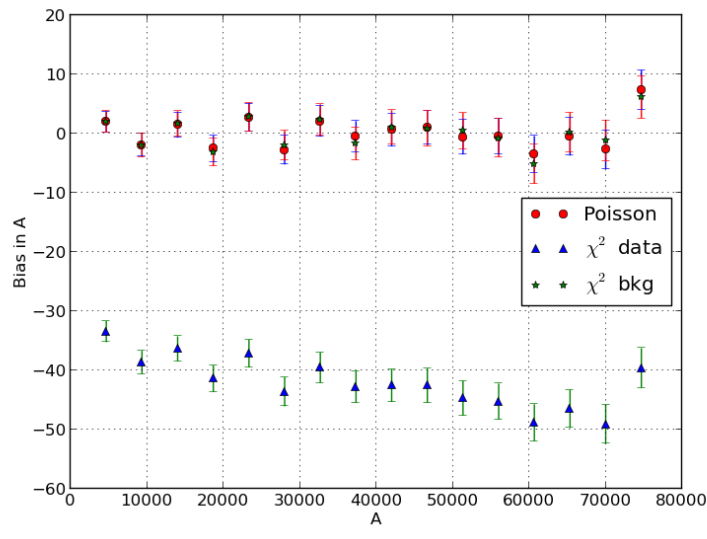

(a)

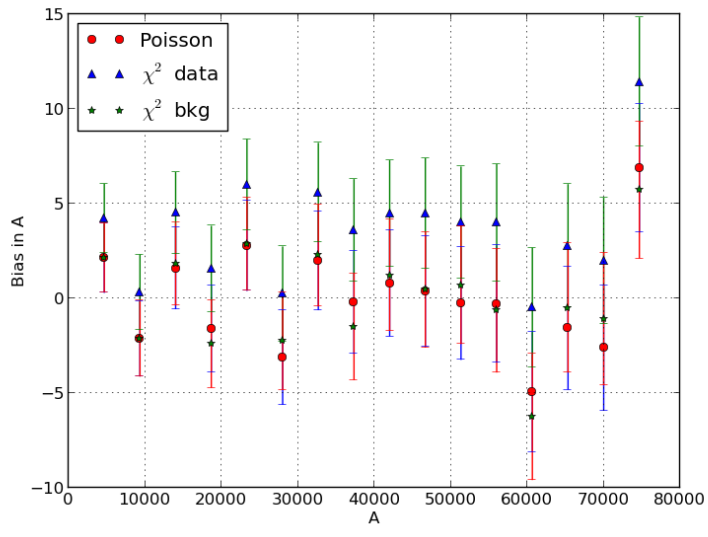

(b)

Fig. 1.- Figure 1a shows the bias in $A$ of the 1-parameter model, which fits only for $A$, as a function of $A$. Figure $1 \mathrm{~b}$ shows the bias in $A$ as a function of $A$ in the 2-parameter model, which fits for both $A$ and $B$.

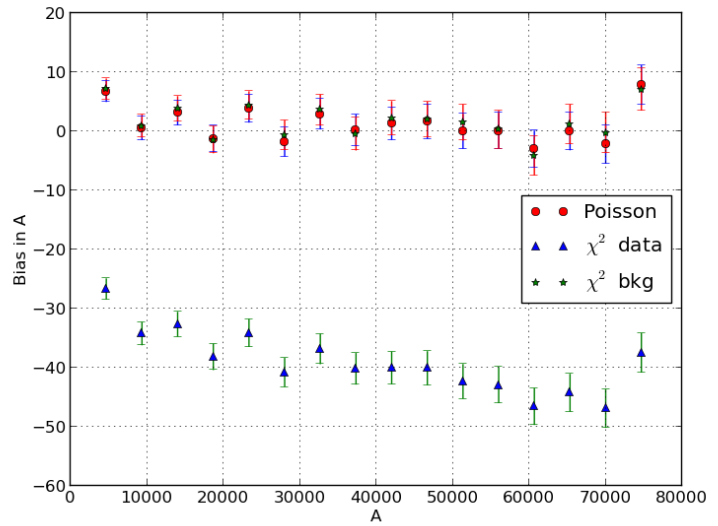

(a)

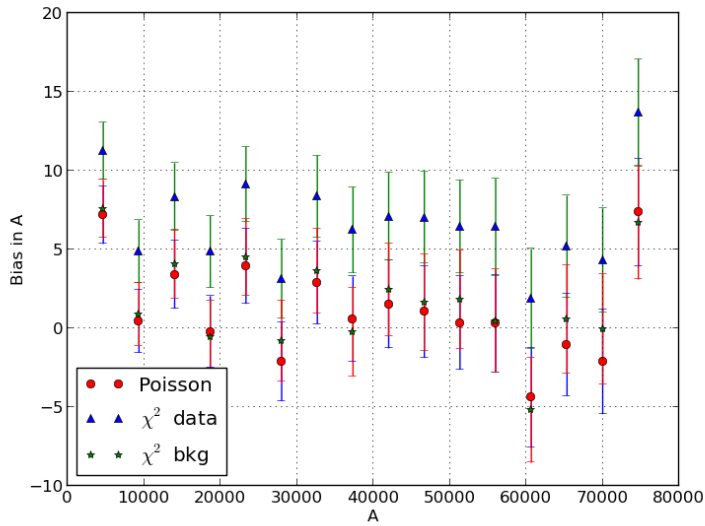

(b)

Fig. 2.- Figure 2a shows the bias in $A$ as a function of $A$ for the 3-parameter model, which fits for $A, s_{x}$, and $s_{y}$. Figure $2 \mathrm{~b}$ shows the same, for the 4-parameter model, which encompasses fits for $A, B, s_{x}$, and $s_{y}$. The series are labeled as above. 


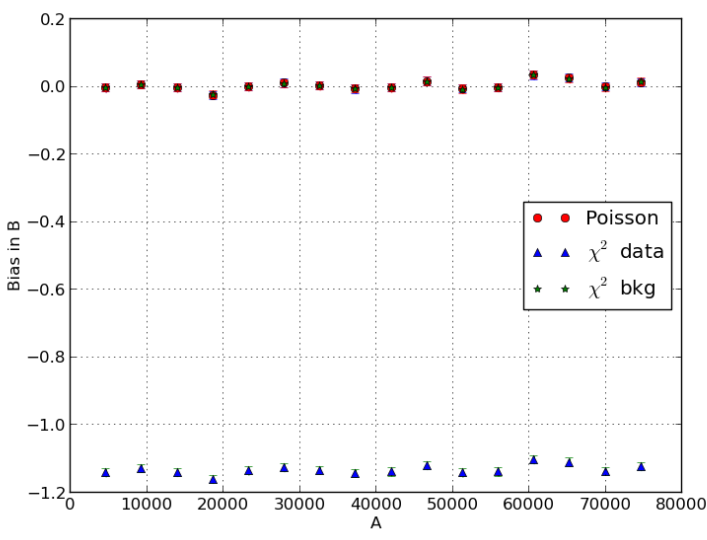

(a)

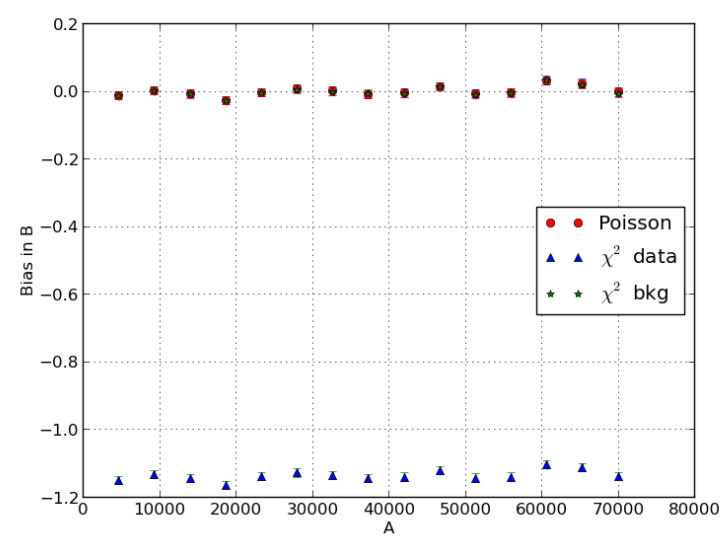

(b)

Fig. 3.- Figures. 3a and 3b show the bias in parameter $B$ as a function of $A$ for the $n=2$ and $n=4$ cases, respectively. The 2 - and 4 -parameter systems are the only sets that fit for $B$, and hence then only systems in which $B$ is varied in the fitting process. Here, again, NTRIALS=10,000 and the true value of $B$ is used.

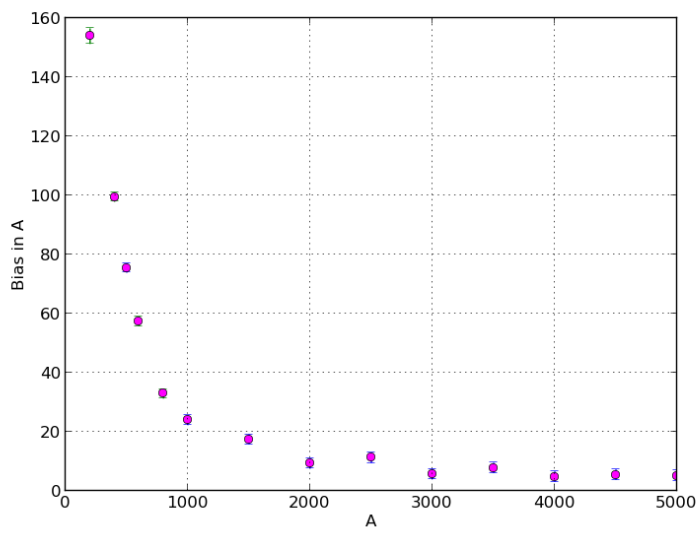

(a)

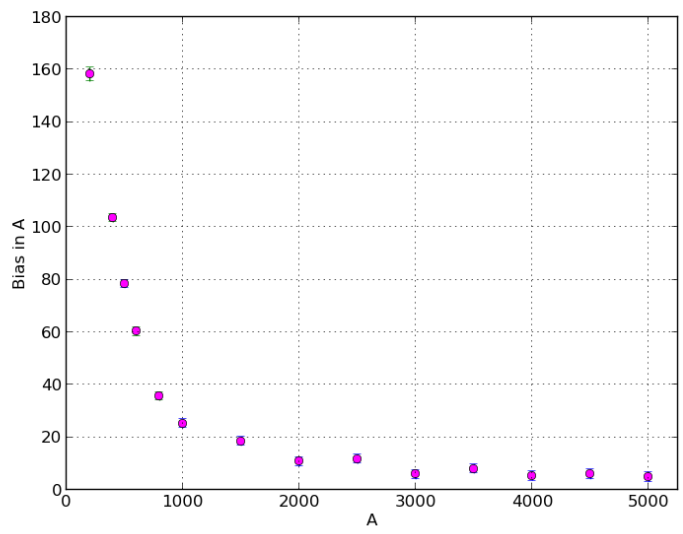

(b)

Fig. 4.- Figure 4a shows the small signal region of the Poisson series for the 3-parameter system. Figure $4 \mathrm{~b}$ shows the same series in the 4 -parameter system. Both fits were constructed with NTRIALS $=10,000$ and using the true value of $B$ without deviation $\left(\sigma_{p}=-1\right)$. 
level (parameter $B$ ) in calculating the fit (refer to equation 8). In these plots, we set $A$ and $B$ to their true values (1062 and 742, respectively) and investigate how the bias in fitting these varies over a range of background estimates. The graphs indicate that as $B_{P}$ approaches the true value of $B$, the biases in both $A$ and $B$ approach zero. For both 5a and 5b we provide linear fits of the bias curves. In $5 \mathrm{a}$, we note that the curve displays non-linear features, but for the small range we are considering, a linear approximation suffices. The function describing the linear fit is given by

$$
f(x)=-37.8 x+28246
$$

. The important feature of this equation is the slope, which describes the ratio of error between $B_{P}$ and $A$. With magnitude of approximately 1:40, we determine that an error of 0.1 in $B_{P}$ corresponds to an error of approximately 4 in $A$. Understanding this effect enables a correction formula to be used, if the error in $B_{P}$ is known or can be estimated.

In Figure 5b, the linear fit is given by

$$
f(x)=0.986 x-731.3
$$

and we are again primarily concerned with the slope. We expect the slope to be unity, as forcing a prior on $B$ intrinsically changes the value computed for $B$, since the computer must use the prior. The difference between whatever value is used for $B_{P}$ and the true value of $B$ will be exactly the bias in $B$. We conclude that the biases in both $A$ and $B$ depend on $B_{P}$ and vanishl as the true background level is approached.

\subsection{Variance}

The variance of a parameter is an indication of the amount of fluctuation between trials, or "spread" of the data. The variance in $A$ is defined mathematically as the RMS value for each data point. The RMS is given by

$$
\sqrt{\Sigma \frac{x_{i}^{2}}{\text { ntrial }}-\bar{x}^{2}}
$$

where $x_{i}$ represents once instance of the bias, or difference between the true value of a parameter and its computed value, $\bar{x}$ represents the average value of the bias, and ntrial is the number of successful fits. Figure 6 shows the variance in $A$ divided by $A$, or normalized variance, as a function of $A$ for the $n=1$ and $n=2$ cases, and Figure 7 shows the same for the $n=3$ and $n=4$ cases. While the variance curves are nearly identical for all fits and parameter systems, plotting a subtracted curve reveals a difference between the fits. While the differences are statistically significant, they are negligible, occurring on a scale 3 orders of magnitude smaller than scale on which the curves are presented.

A peak value ranging between 0.35 and 0.40 occurs at the first sampled point for each fit in each parameter system, whereafter the variance decays with increased signal strength. Less fluctuation 
with improved signal strength is anticipated, though the variance decays much more slowly than the bias.

Figures $8 \mathrm{a}$ and $8 \mathrm{~b}$ provide additional details on the fits. Figure 8a highlights the divergence of the $\chi^{2}$ background series from the Poisson likelihood and $\chi^{2}$ data series as signal strength is increased to very high values. This plot is logarithmic in the $x$-axis, and shows the behavior of all three fits for the $n=3$ case for signal strengths up to $A=5 \times 10^{7}$. In Figure 8 a, the background level is set at $B=742$. In Figure $8 \mathrm{~b}$, we examine a region with high signal strength $\left(A=10^{7}\right)$ and low bias, which serves to contrast the previous scenarios, in which the signal-to-bias ratio was much lower. If we compare this RMS plot to the one constructed for the $n=3$ case in Figure 7 , we see that the high signal case does not show a decay and displays a discrepancy between the three fitting methods, most noticeably in the $\chi^{2}$ background series. In the typical signal range used in earlier figures, we see that the RMSs of the three fits align nearly identically, and decay toward zero. We note that in this case (figures $8(\mathrm{a})$ and $8(\mathrm{~b})$ ), the true value of $B$ was set to 742 . In the high signal series, the RMS is much larger, meaning there is a much higher variance among the data points, and that the fits behave distinctly. The Poisson and $\chi^{2}$ with data error series do not align to within minute discrepancy, as is the case in the normal signal range, and the $\chi^{2}$ with background error series demonstrates a much higher variance over the given range of background levels. In figures 6 and 7 , we varied $A$ and kept $B$ at its true value, whereas in Figure $8 \mathrm{~b}$, the background level is varied over a range of small values, and the signal is high. Despite the different plotting schemes, it is easy to see that the fits behave differently in the two situations. We see that the $\chi^{2}$ fit with background error behaves most unfavorably of the three under these conditions, and that the Poisson fit works just as well as, but not significantly better than, the traditional $\chi^{2}$ fitting method with data error.

\subsection{Error Estimate}

In addition, we collected measurements of the error estimate of each fit as a function of $A$. The fit estimates parameter errors based on the input image data. More generally, each parameter in the likelihood or minimization routine has an associated uncertainty. The error estimate is calculated by summing the square of the fit error on each parameter for each iteration, dividing by the number of successful fits (ntrials), and taking the square root of that value. The error estimate is based on the errors of each input to the fitting routines; it is not related to the true values. The square of the error associated with each of $A, B, s_{x}$, and $s_{y}$ is recorded along the diagonal of a covariance matrix. The total error of the fit is calculated from the errors on each relevant constituent in the matrix ( $A$ and $B$ for $n=2$, and so forth). This value is recorded for each data point. Figures $9(n=1,2$ cases $)$ and 10 ( $n=3,4$ cases $)$ show the error estimate versus $A$ for each parameter system. The most noticeable observation is the constancy of the $\chi^{2}$ background series in all four cases. While the error estimates in the Poisson and data error series increase with signal strength, the background error series remains fixed at an error estimate lower than is ever 


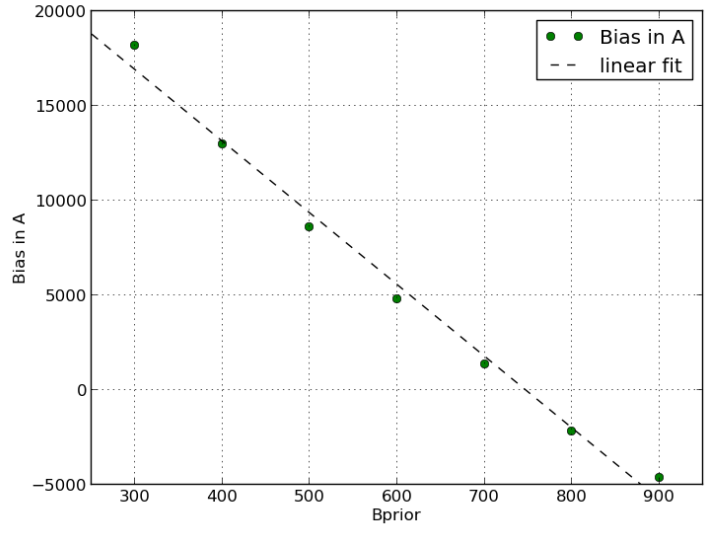

(a)

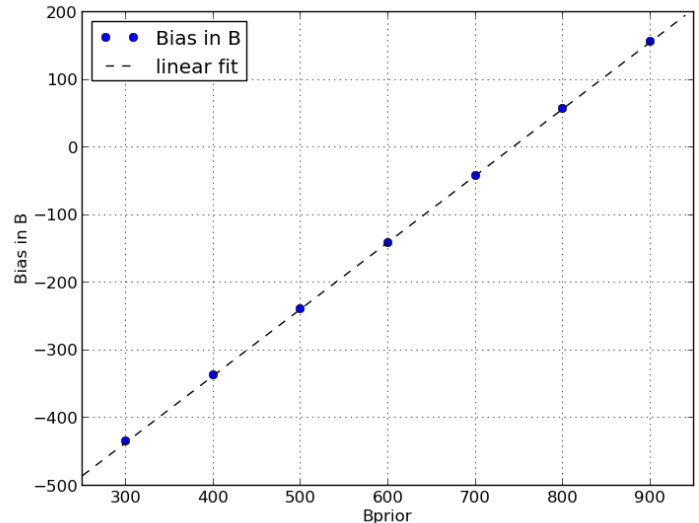

(b)

Fig. 5.- Figure 5a shows the bias in $\mathrm{A}$ as a function of $B_{P}$. Figure $5 \mathrm{~b}$ shows the bias in $B$ versus $B_{P}$. In both cases, fixed values of parameters $A$ and $B$ were set; $A=1062, B=742$. These graphs were generated with the Poisson fit on the 4-parameter systems, with NTRIALS $=10,000$ and using small deviations from the true value of $B\left(\sigma_{p}=0.1\right)$. The effect of modulating $B_{P}$ in the $n=1,2$, and 3 -cases is similar, so we include only the $n=4$ case as an example.

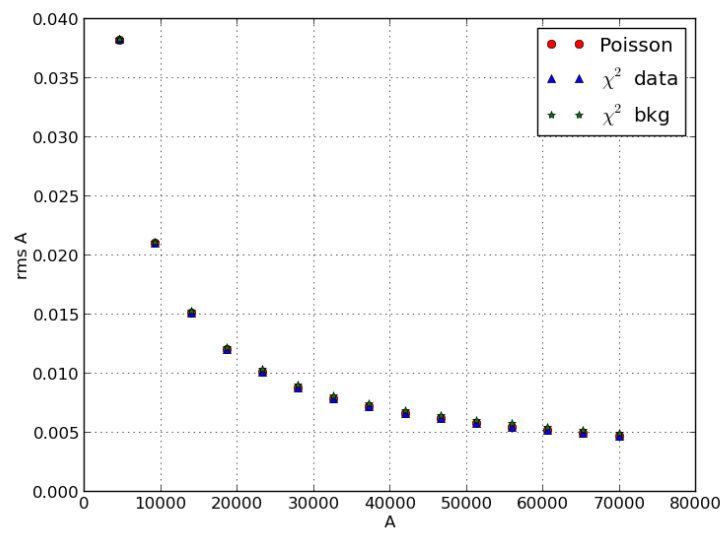

(a)

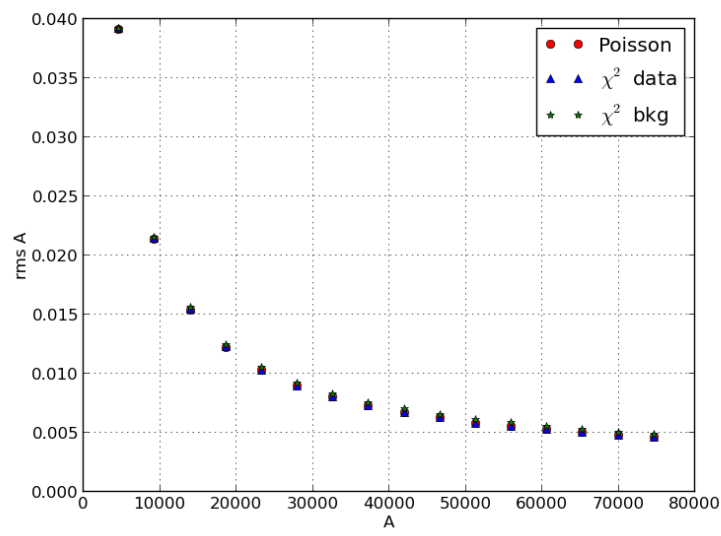

(b)

Fig. 6.- Figure 6a shows the normalized RMS of $A$ as a function of $A$ for the $n=1$ case; Figure $6 \mathrm{~b}$ shows the same for the $n=2$ case. Fits were constructed with NTRIALS $=10,000$ and using the true value of $B$. 


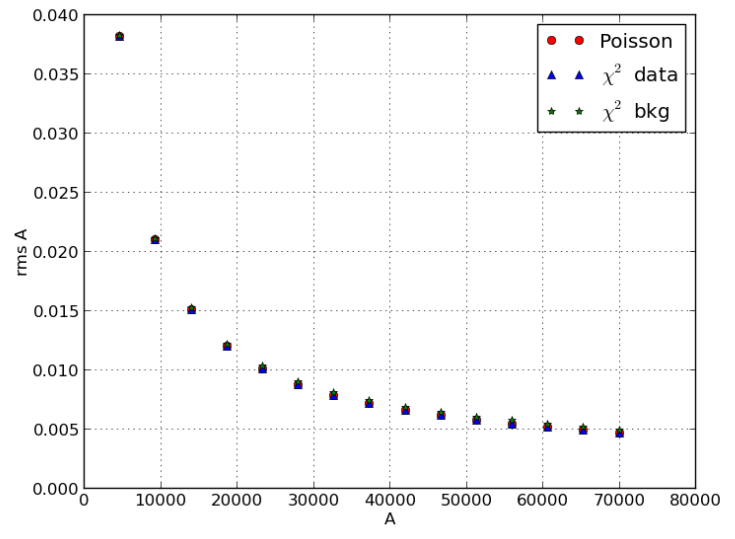

(a)

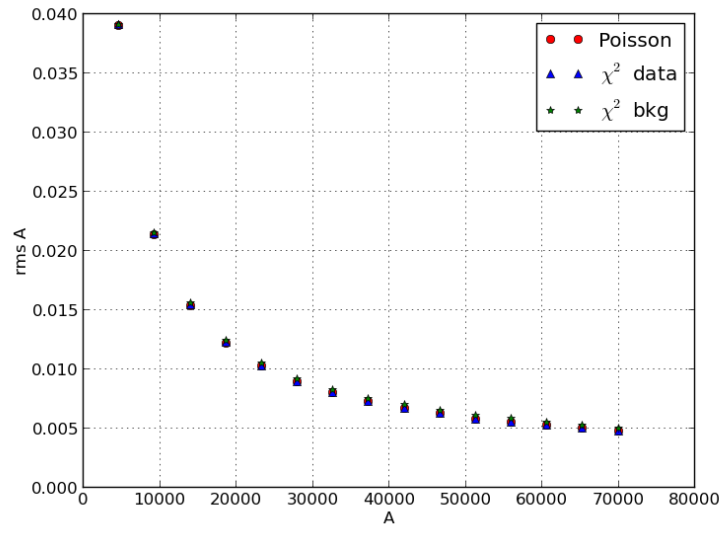

(b)

Fig. 7.- Figure 7a shows the normalized RMS of $A$ as a function of $A$ for the $n=3$ case; Figure $7 \mathrm{~b}$ shows the same for the $n=4$ case. Fits were constructed with NTRIALS $=10,000$ and using the true value of $B$.

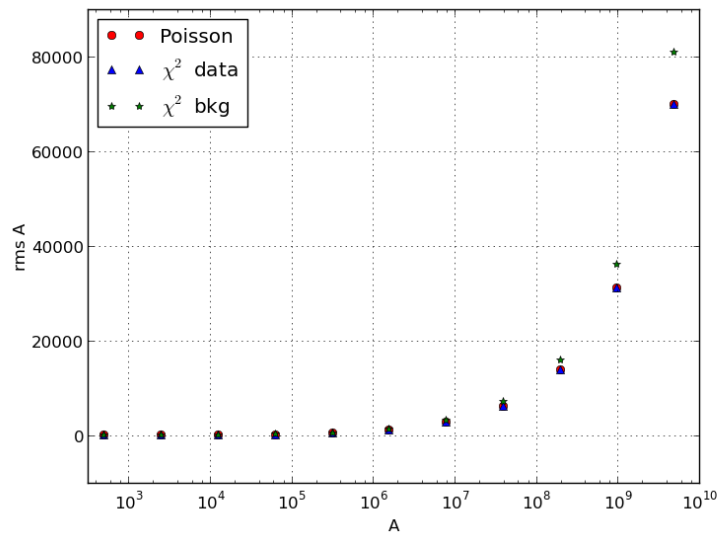

(a)

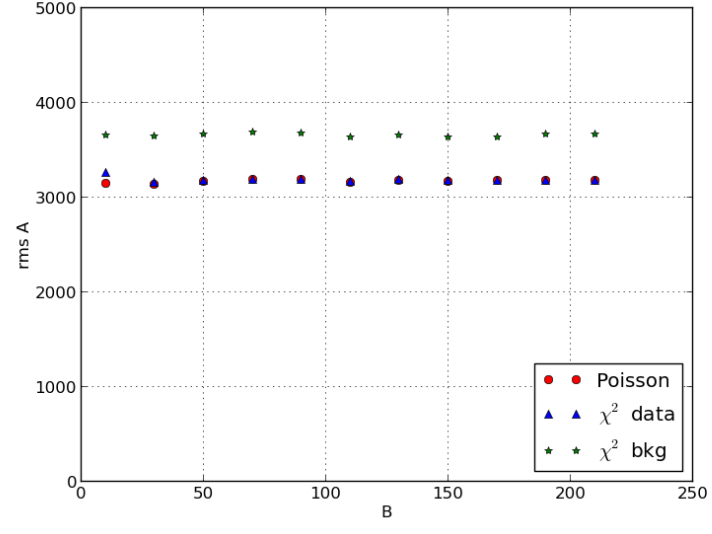

(b)

Fig. 8. - Figure 8a shows the escalation of the divergence of the $\chi^{2}$ background series from the other two as a function of signal strength. This is a semi-log plot, showing signal strength over the range 500 to $5 \times 10^{7}$. In Figure $8 \mathrm{~b}$ we show the RMS of $A$ as a function of $B$ for each of the three fits in the $n=3$ case. Here, we have forced the signal to remain at a constant high value by setting the true value of $A$ to $10^{7}$ and looped over small background values of $B$. This plot demonstrates that a discrepancy between the variances of three fits becomes noticeable with high signal and low-background levels. The $\chi^{2}$ background RMS diverges from the other two series in this case. The fits were computed with NTRIALS $=10,000$ and using the true value of $B$. 
reached by either of the other series because the error in the $\chi^{2}$ background series does not depend on signal strength. The explanation for this behavior is that the $\chi^{2}$ background fit estimates the error values incorrectly, except when $A=0$, highlighting an obvious problem in this choice of fit. This explanation is substantiated by the series that show the RMS, without normalization by $A$, as dashed curves next to the error estimate series for each fit. In all of these figures, we see that the RMS of the $\chi^{2}$ background series follows a curve very similar to those of the Poisson and $\chi^{2}$ data fits, whereas the actual error estimate is low and does not display any curvature. This shows that the $\chi^{2}$ background series does not compute the error correctly. If it did, its error estimate and RMS would be nearly the same, as they are in the case of the other two fits. The $\chi^{2}$ background fit computes the error incorrectly because the fit uses a background estimate $\sigma_{i}$ (see $\S 2$ ), which results in a flat error estimate despite increased signal. The error estimate should increase with increased signal, a behavior that the Poisson and $\chi^{2}$ data fits both demonstrate.

\subsection{Discussion}

The standard statistical indicators examined here - bias, variance, and error estimate - show that the fit based on Poisson statistics is a superior method to either of the $\chi^{2}$ fits. In measures of bias, the Poisson fit yields biases consistent with zero for both $A$ and $B$ as a function of signal strength when $s_{x}$ and $s_{y}$ are fit. This is not true of the $\chi^{2}$ data method. In measures of variance, all three fits behave similarly, with the exception of the high signal range, in which the $\chi^{2}$ background fit diverges and shows a higher variance than the other two. In measures of the error estimate, the $\chi^{2}$ background fit fails to fit the errors, and hence does not provide any reliable measure. The Poisson and $\chi^{2}$ data fits show the same behavior. From these graphical analyses, we glean that the fit based on Poisson statistics is more efficient and reliable, as it succeeds in every one of these statistical categories, unlike either of the $\chi^{2}$ methods. We conclude that the Poisson method is best for fitting the PSF.

\section{Sensitivity to Data and Error Model}

Having analyzed the Poisson fitting method under typical conditions alongside that of the $\chi^{2}$ methods and concluded that it is the best fitting routine, we include more detailed analysis of the Poisson likelihood fitting method and highlight its sensitivities. We concentrate on the behavior of the Poisson likelihood method, but note that the other methods should behave similarly.

\subsection{Sensitivity to PSF}

Figure 11 maps the behavior of the Poisson fit with the true value of $A$ held at its standard value of 1062 . but with the true value of $B$ set to a series of values incremented over multiplication 


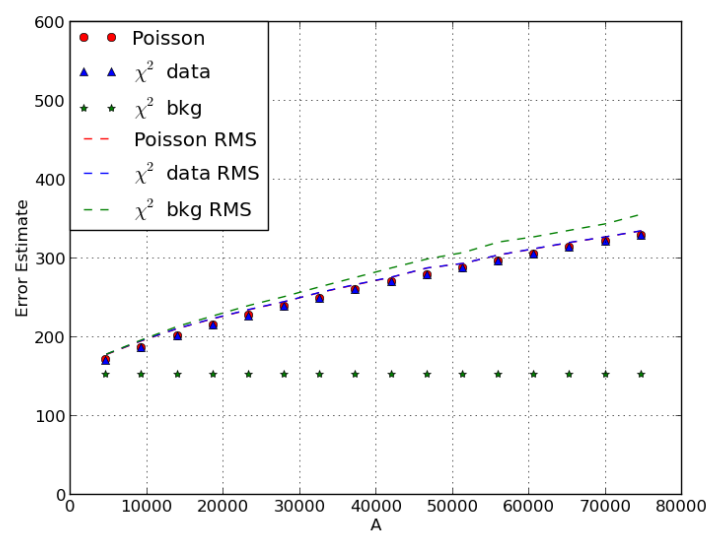

(a)

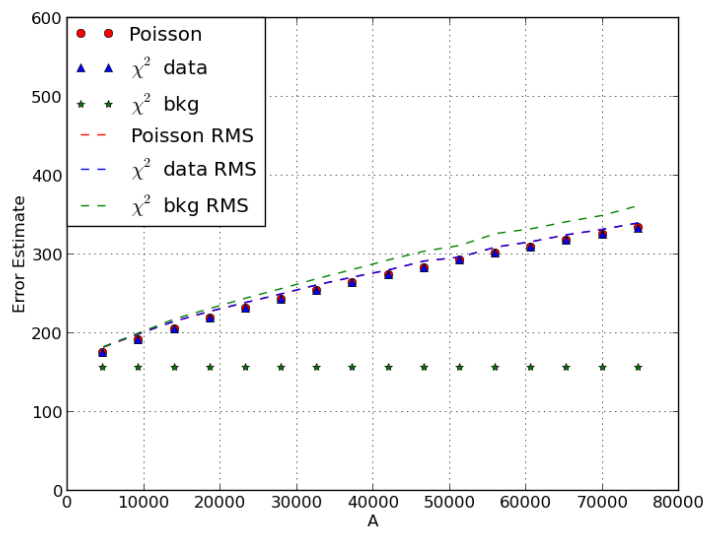

(b)

Fig. 9.- These figures show the error estimate of each fit in solid markers and the RMS of the corresponding fit in a dashed line of the same color. Figure $9 \mathrm{a}$ is the $n=1$ case, Figure $9 \mathrm{~b}$ is the $n=2$ case. The misalignment of the $\chi^{2}$ background fit's error estimate with its RMS is indication that it is computing errors incorrectly. Fits were constructed with NTRIALS=10,000 and using the true value of $B$.

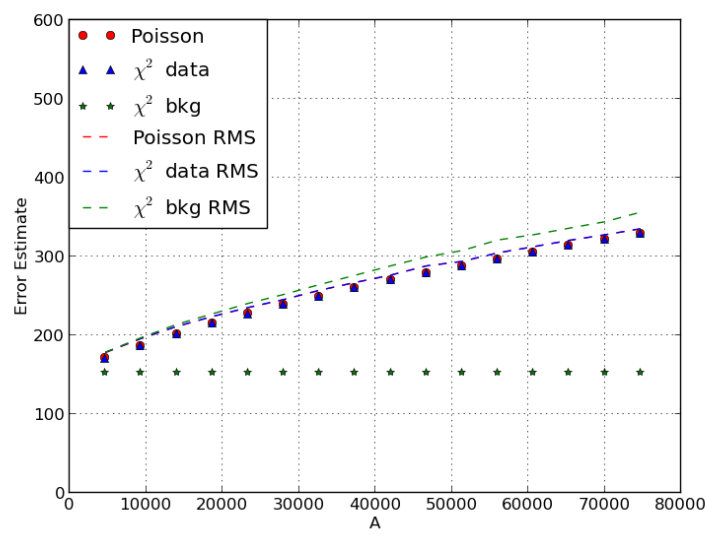

(a)

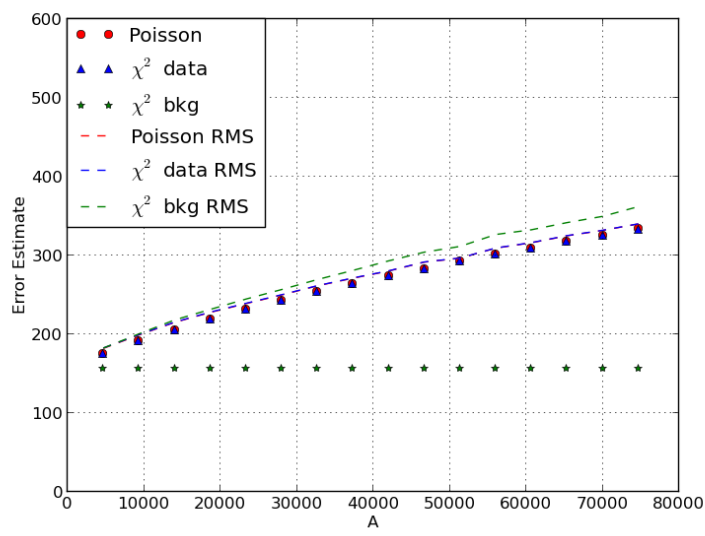

(b)

Fig. 10. - The error estimate of each fit is shown in solid markers, and the RMS of the corresponding fit in a dashed line of the same color. Figure 10a is the $n=3$ case, Figure 10b is the $n=4$ case. The misalignment of the $\chi^{2}$ background fit's error estimate with its RMS is evident in these two cases as well. Fits were constructed with NTRIALS $=10,000$ and using the true value of $B$. 
by two, beginning with $B=371$ and extended to $B=5936$. We plot the bias in $A$ for each of these $B$ values as a function of the term

$$
\frac{\delta s x^{2}+\delta s y^{2}}{\text { ntrial }}
$$

which is a composition of three variables that are tracked in the construction of the fit: ntrial, which logs the number of fits that succeed, and $\delta s x$ and $\delta s y$, which are the error estimates of the position. Note that displacement can only be positive, since the center is taken at $s_{x}=s_{y}=0$.

Figure 11a shows the bias in $A$ as a function of signal strength for a series of $B$ background levels ranging from half of the true $B$ value to $8 \times$ the true $B$ value. This is shown in the small signal region $A=500$ to $A=5000$. We see negative effects here for the higher $B$ levels, and note that although the series decay for every $B$ value, the bias is larger when $B$ is large. Figure $11 \mathrm{~b}$ indicates that as the radial error estimate increases from zero, the bias in $A$ increases rapidly. Because an error in centering the PSF will affect the bias, the dependence on the error estimate is expected. The figure also demonstrates that with higher background level, the bias is stronger and increases more rapidly.

Two more behaviors we investigate for the Poisson likelihood fit are shown in Figure 14. The first, Figure 14a, is relevant to measures of sensitivity to the PSF. The bias's response to a $10 \%$ misalignment error between the standard deviation of the data curve and the standard deviation of the PSF is shown in Figure 14a. We have forced the incongruity by setting the ratio of the standard deviations (see explanation of Figure 12) to 1.1 by holding $\sigma_{\text {data }}$ at 1.7303 pixels, $10 \%$ wider than $\sigma_{p s f}$. The plot demonstrates that this has a drastic effect on the bias in signal, and that the negative effect worsens with increased signal strength. Note that Figure 14a shows signal strength $A$ on a log scale, extending into the very high signal region. Note also that the magnitude of the bias in $A$, rather than the true bias in $A$, is shown; this is for the sake of log-log capability, and all true biases shown in 14a are negative. This simply means that the routine underestimated (rather than overestimated) the value of $A$; the size of the discrepancy is most important. The curve in $14 \mathrm{a}$ is non-linear, but a correction formula could still be computed and would be a powerful tool for correcting the biasing if a misalignment error were known preemptively. It is often the case, however, that a misalignment error is not known ahead of time.

We explore the Poisson fit's sensitivity to the wrong PSF in Figure 12, where we show the bias in $A$ as a function of a ratio between two standard deviations, or widths of the Gaussians, from the fictitious data set and the fake PSF, respectively. The one representing the fake data set is called $\sigma_{\text {data }}$, and the one representing the fake fit is called $\sigma_{p s f}$. In the standard fits, this ratio is taken to be to unity. In this plot, we vary the ratio between approximately 0.5 and 1.5 by changing the standard deviation of the fake data only; the sigma value of the fake PSF is held constantly at 1.573 pixels. The behavior of the Poisson fit over this range is shown for the $n=1,2,3$, and 4 cases in Figure 12. Note first that where the ratio approaches unity, the signal bias approaches zero in all cases. We see a slightly curved, downward trend as the ratio increases in all four parameter 


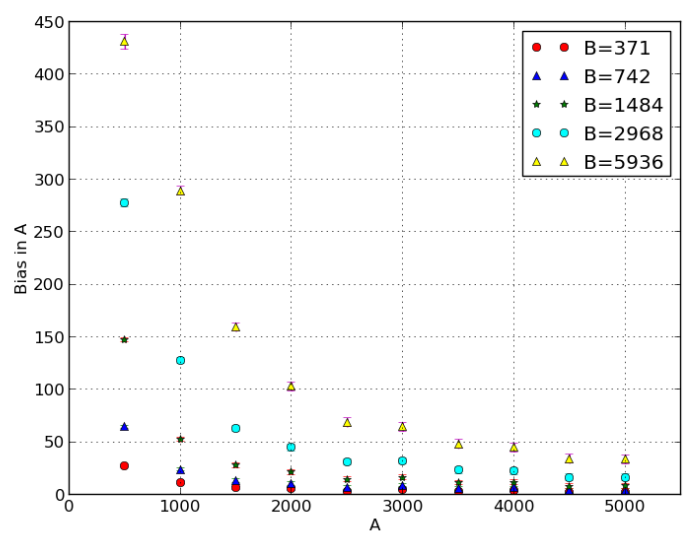

(a)

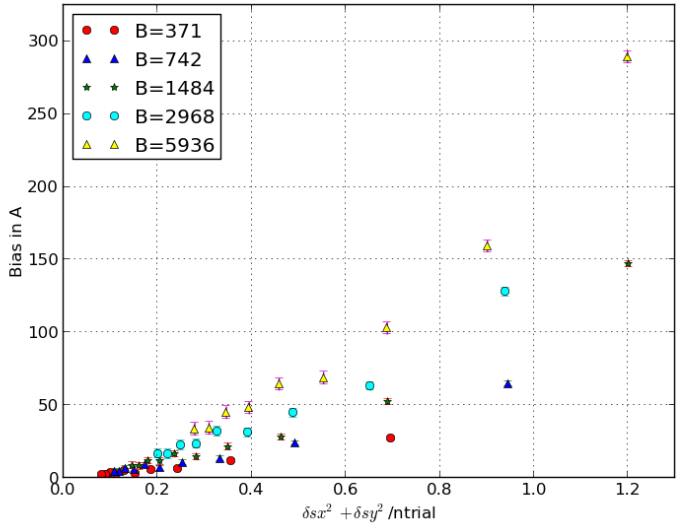

(b)

Fig. 11.- Figure 11a shows the bias in $A$ as a function of signal strength, for background levels listed. Figure $11 \mathrm{~b}$ shows the bias in $A$ the same set of background levels as function of the term described in equation 11, which describes the radial displacement of the PSF from the true center. The background levels for each series are indicated on the legend. All series in these figures are products of the Poisson fit for the $n=4$ case. Figures were computed with the true value of $B$ and NTRIALS $=10,000$. Error bars in this figure are subsumed by the size of the data series.

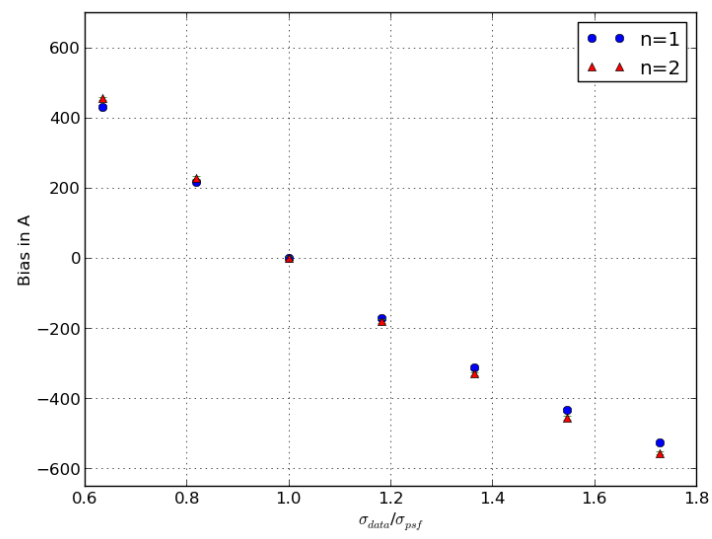

(a)

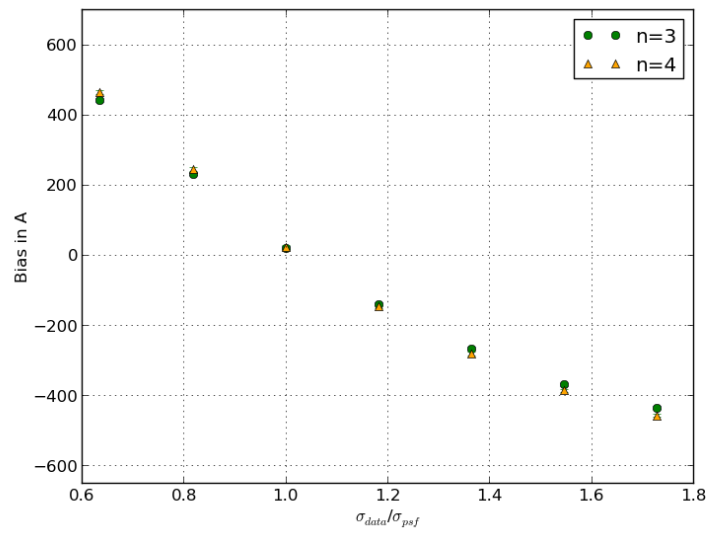

(b)

Fig. 12.- Figure 12a shows the bias in $A$ as a function of the ratio of standard deviations $\sigma_{\text {data }}$ and $\sigma_{p s f}$ for the $n=1$ and $n=2$ cases with the Poisson fit. Figure $12 \mathrm{~b}$ shows the same for the $n=3$ and $n=4$ cases. At unity in both figures, the bias in $A$ is approximately zero. Figures were generated using the true value of $B$ and NTRIALS $=10,000$. 


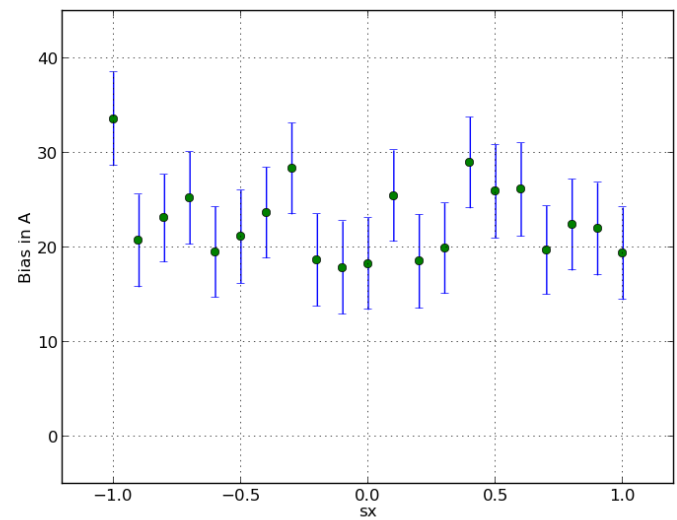

(a)

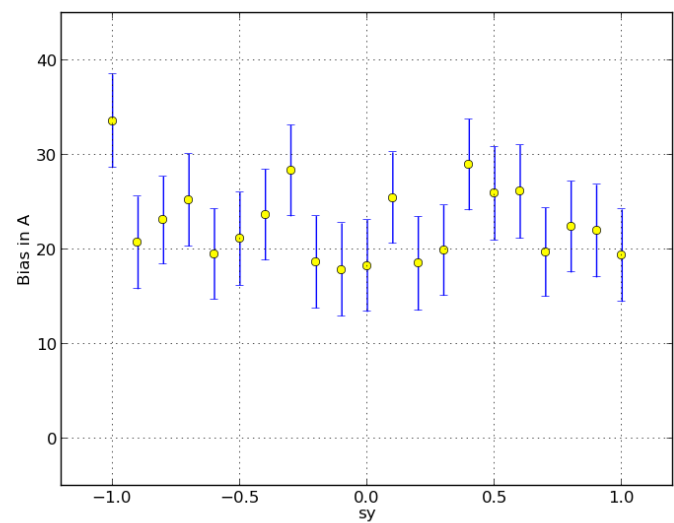

(b)

Fig. 13.- Figure 13a shows the bias in $A$ as a function of $s_{x}$, one of two symmetric position parameters. Figure $13 \mathrm{~b}$ plots the bias in $A$ as a function of the other position parameter, $s_{y}$. The graphs are identical, as anticipated. We show only the $n=3$ case as demonstration. Values were computed with NTRIALS $=10,000$ and using the true value of $B$.

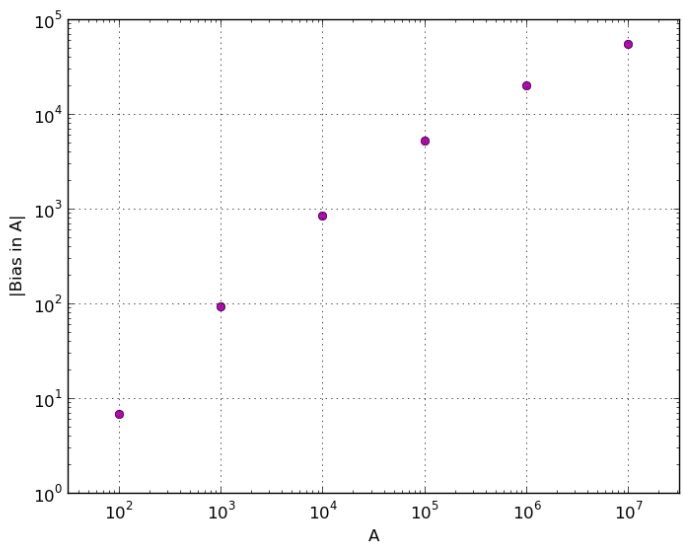

(a)

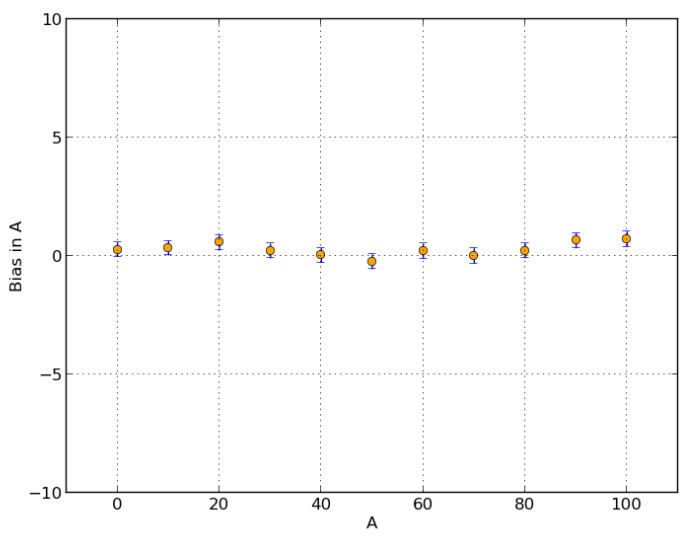

(b)

Fig. 14. - Figure 14a shows the bias in $A$ as a function of signal strength under the constraint of a $10 \%$ error in the data-PSF alignment for the $n=1$ parameter system. The scale is log-log, showing a large signal range, and the magnitude in bias is shown for the sake of logarithmic plotting. The all of the true bias values in this figure are negative. The fit in Figure 14b was constructed using and Poissonian, rather than Gaussian, noise generator. It shows the bias in $A$ as a function of $A$ in a very small signal region, with the background level set at a constant low value of $B=3$. Both fits were computed with NTRIALS $=10,000$ and using the true value of $B$. 
systems, indicating that where the standard deviation of the fake data is less than that of the fake PSF, there is positive biasing, and as $\sigma_{d a t a}$ increases compared to $\sigma_{p s f}$ and surpasses it, the biasing shifts negatively and increases in severity. In all four cases, the biasing appears nearly antisymmetric across the unity point, with similar magnitude as the ratio is shifted in either direction away from 1 . We note that the $n=1$ and $n=3$ cases appear nearly identical, as well as the $n=2$ and $n=4$ cases. This is explicable in terms of whether or not the background level is being fit.

\subsection{Sensitivity to Position}

Another important aspect of the fitting response is the interpolation of the PSF. As a test of PSF interpolation, we include measures of the bias in $A$ as a function of position parameters $s_{x}$ and $s_{y}$ for the relevant parameter systems (3 and 4$)$ in Figure 13. Parameters $s_{x}$ and $s_{y}$ are symmetric measures of the horizontal and vertical displacement of the PSF. We vary $s_{x}\left(s_{y}\right)$ over a range of -1.0 to 1.0, imitating at most a one-pixel offset to the right or left. What we find is that the bias in $A$ does not change from its value at $s_{x}=s_{y}=0$, which we had already known from earlier plots, as a function of either position parameter- the bias of approximately 20 remains constant when the center of the PSF is shifted up to one pixel in either direction. The bias in $A$ looks the same in Figures $13 \mathrm{a}$ and $13 \mathrm{~b}$, as $s_{x}$ and $s_{y}$ are symmetric and control the same aspect of the PSF's location.

\subsection{Sensitivity to Error Model}

The final figure, Figure 14b, reflects the Poisson likelihood fit's response to the error model. Figure 14b shows a first glimpse of the effects of altering the noise generator. For every other data set presented here, we have added noise taken from a Gaussian distribution to the signals. In 14b, we take the noise from a Poisson distribution instead. First analysis of a very small signal region, with low background, reveals that changing from a Gaussian to Poissonian generator produces no adverse effects in the bias in $A$, at least to within two standard deviations. Consistency with zero bias could be shown definitively with an NTRIALS value of 100,000 rather than 10,000, but this is computationally taxing, and we suspect that the noise generator will not negatively affect the biasing.

\section{Conclusions}

We have demonstrated that the fitting method based on Poisson statistics works as well as or better than the $\chi^{2}$ methods. It is efficient in more circumstances than either of the other two methods, and shows statistical robustness in measurements of biasing, the variance, and the error estimate. The Poisson fit is highly effective by all three of these standards, whereas neither the $\chi^{2}$ data nor $\chi^{2}$ background fit is optimally functional in all of these respects. We have shown that the 
Poisson fit is susceptible to distortions in the small signal region, when the background estimate is off, or when the centering of the PSF is off. We suggest that the fitting method based on Poisson statistics would be a better substitute for traditional $\chi^{2}$ fitting methods, and advocate its use in PSF photometry.

Further work could include deeper analysis of the quirks of the Poisson likelihood fit, finding correction formulas for well-understood biasing behaviors, and more thorough investigation into the benefits of a Poissonian noise generator.

\section{A. Linearized Equations}

The maximum likelihood can be found by setting the derivatives of its logarithm to zero

$$
-2 \frac{\partial \ln \mathcal{L}}{\partial \alpha_{k}}=0
$$

and solving for the four fit parameters $\alpha_{k}=A, B, s_{x}, s_{y}$. We solve the equations by linearization and iteration. This assumes that we have starting values that are sufficiently close to the solution that the process converges to the proper solution.

The logarithm of the likelihood can be expanded in a Taylor series as follows:

$$
\ln \mathcal{L}\left(\alpha_{m}\right)=\ln \mathcal{L}(\alpha)+\frac{\partial \ln \mathcal{L}}{\partial \alpha_{k}}(\alpha) \delta \alpha_{k}+\frac{1}{2} \frac{\partial^{2} \ln \mathcal{L}}{\partial \alpha_{k} \partial \alpha_{l}}(\alpha) \delta \alpha_{k} \delta \alpha_{l}
$$

where $\alpha_{m}$ represents the parameters at maximum and the sum over $l$ and $m$ is implied. Since the likelihood only depends implicitly on the parameters $\alpha_{k}$ through the function $\bar{n}_{i}\left(\alpha_{k}\right)$, the derivatives can be written as

$$
\frac{\partial \ln \mathcal{L}}{\partial \alpha_{k}}=\frac{d \ln \mathcal{L}}{d \bar{n}_{i}} \frac{\partial \bar{n}_{i}}{\partial \alpha_{k}}
$$

and

$$
\frac{\partial^{2} \ln \mathcal{L}}{\partial \alpha_{l} \partial \alpha_{k}}=\frac{d^{2} \ln \mathcal{L}}{d \bar{n}_{i}{ }^{2}} \frac{\partial \bar{n}_{i}}{\partial \alpha_{k}} \frac{\partial \bar{n}_{i}}{\partial \alpha_{l}}+\frac{d \ln \mathcal{L}}{d \bar{n}_{i}} \frac{\partial^{2} \bar{n}_{i}}{\partial \alpha_{k} \partial \alpha_{l}} .
$$

and the sum over $i$ (all the pixels) is implied. The explicit formulae for the derivatives are:

$$
\begin{aligned}
& \frac{\partial \bar{n}_{i}}{\partial A}=P\left(x_{i}-s_{x}, y_{i}-s_{y}\right) \\
& \frac{\partial \bar{n}_{i}}{\partial B}=1 \\
& \frac{\partial \bar{n}_{i}}{\partial s_{x}}=-\frac{\partial P}{\partial x}\left(x_{i}-s_{x}, y_{i}-s_{y}\right), \\
& \frac{\partial \bar{n}_{i}}{\partial s_{y}}=-\frac{\partial P}{\partial y}\left(x_{i}-s_{x}, y_{i}-s_{y}\right) .
\end{aligned}
$$




\section{B. Solution of the Equations}

Near the peak of the likelihood the second derivatives of the logarithm of the likelihood should be a positive definite, symmetric matrix. The first term in Equation (A4) has this property but the second term, in general, does not. We find that including that term does not significantly improve the rate of convergence and, in fact, increase the number of cases that fail to converge. For that reason, we determine the step to be taken at each iteration by solving the linear equations

$$
\sum_{i=1}^{N^{2}}\left(\sum_{l=1}^{4} \frac{d^{2} \ln \mathcal{L}}{d \bar{n}_{i}{ }^{2}} \frac{\partial \bar{n}_{i}}{\partial \alpha_{k}} \frac{\partial \bar{n}_{i}}{\partial \alpha_{l}}+\frac{d \ln \mathcal{L}}{d \bar{n}_{i}} \frac{\partial \bar{n}_{i}}{\partial \alpha_{k}}\right)=0
$$

where the sum over $N^{2}$ pixels and the 4 fit parameters is now shown explicitly. The derivatives are evaluated as the current value of the parameters $\alpha_{k}$ and the solution for the $\delta \alpha_{k}$ are the incremental changes to the current values.

Initial values are taken to be $s_{x}=s_{y}=0$ for the position. The initial background level $(B)$ is given to the fit. In practice, it might be determined by a non-local analysis of the image background. The initial value for the signal $(A)$ is estimated from the pixel data near the assumed peak $\left(s_{x}=s_{y}=0\right)$.

This solution of Equation (B1) does not guarantee that the updated parameters will result in an increased value of the likelihood function, but in most cases taking a smaller step will result in an increase. For each iteration, the likelihood function is checked to see if the new values of the $\alpha_{k}$ result in an increase. If so, the step is accepted and the fit proceeds with the next iteration. If not, the step size is decreased by a factor of two, and the likelihood functions is checked with the reduced step size. If the likelihood still does not increase the step size is reduced repeatedly by factors of 2 until the likelihood does increase or until the maximum number of cut steps has been reached. When the maximum number of cut steps (nominally 7 ) has been reached, the fit is flagged as a failure.

The fit is iterated successively until convergence is achieved. The test for convergence is that the change in the logarithm of the likelihood function is small (nominally $1 \times 10^{-4}$ ). If the fit does not converge in a maximum number of iterations (nominally 20), the fit is flagged as a failure.

The major source of difficulty when performing the fit arises from the uncertainty in determining the position when the signal is weak and statistical fluctuations disguise the true position of the peak. In such cases the step size for the position parameters can be very large, but the logic of cutting steps is applied so that the there is a maximum position change for each step (nominally 1 pixel). If successive iterations move the centroid too far (nominally more than 3 pixels), the fit is flagged as a failure. A lesser concern is that a fit iteration may result in $\bar{n}_{i}>0$, a value that can not be accommodated in the Poisson formulation of the likelihood. The cut step mechanism is also applied to force $\bar{n}_{i}>0$ for all i. 